\title{
Gray per Minute
}

National Cancer Institute

\section{Source}

National Cancer Institute. Gray per Minute. NCI Thesaurus. Code C158296.

A unit of absorbed radiation dose rate defined as the number of Grays per minute. 